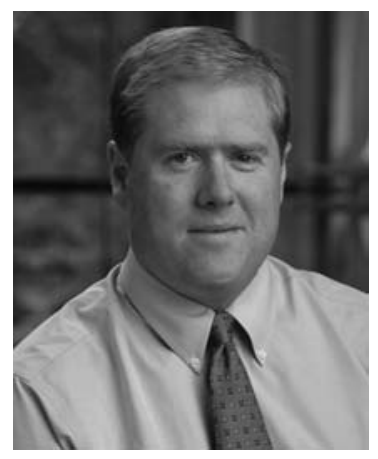

\title{
A Conversation with Sean Morrison
}

\author{
INTERVIEWER: MiRNA KVAJO \\ Scientific Editor, Cell
}

Sean Morrison is Mary McDermott Cook Chair in Pediatric Genetics and the Kathryne and Gene Bishop Distinguished Chair in Pediatric Research and Director of the Children's Research Institute at the University of Texas Southwestern Medical Center.

Mirna Kvajo: Stem cells are studied in many contexts, but you're interested in the specific process of self-renewal. Can you tell us about this process and why you decided to study it?

Dr. Morrison: There are many different kinds of stem cells. Self-renewal is the process by which they divide to make more stem cells. This process is fundamental to their ability to persist throughout life in your tissues. We need these in our skin to make new skin cells and in our bone marrow to make new blood cells and bone cells. When I first started my laboratory 17 years ago, I thought the most important question in stem cell biology was to understand the molecular mechanisms that regulate their self-renewal, partly because such self-renewal is necessary for the expansion of stem cells during development during the formation of tissues, but also because it's necessary for the maintenance of stem cells throughout adult life to maintain our tissues.

At the time, there was also a sense that these mechanisms might have something to do with cancer. There were pro-proliferation genes and antiproliferation genes, and it seemed obvious that stem cells would depend on pro-proliferation genes. It turned out to be a lot more interesting than that. As we studied stem cell self-renewal, we learned that the processes were regulated by networks of proto-oncogenes and tumor suppressors. We were bumping into the same genes that regulate cancer cell proliferation. We also learned that stem cell self-renewal is different from the proliferation of restrictive progenitors in the same tissues. We went back and forth between hematopoietic stem cells and neural stem cells, trying to identify key regulators of stem cell self-renewal. We defined this as "gene products that are required for the self-renewal of the stem cells but are not necessarily required for the proliferation of restricted progenitors in the same tissue."

Mirna Kvajo: Do different types of tissue require different regulators?
Dr. Morrison: There are different regulators, but what was more remarkable is that there's a group of regulators that are incredibly conserved across stem cells in many different adult tissues. Those tend to be the genes that get hijacked by cancer cells. Whenever we identified a new regulator of stem cell self-renewal, invariably, it would turn out to be involved in cancer. At least to first approximation, it looks like cancer cells hijack stem cell selfrenewal mechanisms, more so than restricted progenitor proliferation mechanisms. Presumably, there's some advantage to cancer cells hijacking molecular mechanisms that enable a long-term capacity to replicate themselves rather than the limited replicative capacity that you typically see in restricted progenitors.

Mirna Kvajo: What is so special about the self-renewal process that could be so advantageous?

Dr. Morrison: Cancer cells need to replicate themselves extensively. Restricted progenitors often have a limited replicative capacity, whereas stem cells are distinguished from restricted progenitors precisely by their ability to replicate themselves extensively. It means that cancer is a disease of dysregulated self-renewal. The mechanisms that we think of as being so important in cancer biology didn't evolve to regulate the proliferation of cancer cells. These networks of proto-oncogenes and tumor suppressors evolved to regulate normal tissue homeostasis. Their role in cancer reflects the ability of cancer cells to hijack those processes to enable a selfish neoplastic proliferation.

Mirna Kvajo: Do you think that this mechanism is general to most cancers?

Dr. Morrison: Many cancers, but probably not all. For example, Iannis Aifantis and others have shown that there are some mechanisms in T-cell acute lymphoblastic leukemia that are borrowed from restricted T-lineage progenitors. T cells are more like stem cells, though, in the sense that they can go in and out of the cycle for

(C) 2016 Morrison. This article is distributed under the terms of the Creative Commons Attribution-NonCommercial License, which permits reuse and redistribution, except for commercial purposes, provided that the original author and source are credited. 
long periods of time and persist throughout life. In one sense, they're restricted progenitors, but on the other hand, their mode of division is a bit more like that of stem cells.

Mirna Kvajo: You've identified a core of conserved regulators, but you're trying to find others, and you've implicated molecules such as estrogen and leptin in stem cell renewal that might also regulate more general systems. What led you to this line of inquiry?

Dr. Morrison: When we started studying self-renewal mechanisms, there was almost nothing known at a molecular level. If we identified a new transcription factor that was required for hematopoietic stem cell self-renewal or an epigenetic regulator like B-cell-specific Moloney murine leukemia virus integration site 1, it was a Nature paper or a Cell paper. The approaches that we took to identify the genes that were necessary for stem cells' self-renewal are now being used to look for transcription factors, epigenetic regulators, and signaling molecules. We're more interested now in studying aspects of cellular physiology that haven't been studied before in somatic stem cells. Generally, they haven't been studied before because people are used to using 10 million cells, and you can't get 10 million cells if you're studying somatic stem cells. We end up having to scale down assays. As a consequence, we're studying proteostasis in stem cells, we're studying metabolism. We're trying to address the general question of whether stem cells differ from other cells based purely on executive control, where there are a few transcription factors or epigenetic regulators that distinguish stem cells from other cells.

Maybe a lot of the other cellular physiology is the same: the way they segregate chromosomes or metabolize glucose. Maybe most of that is basically the same as in many other cell types. The other possibility is that there are many aspects of cellular physiology that have to be different for a stem cell to create a stable, self-replicating, multipotent state. That's what we're trying to get at. There's an enormous amount of new biology that can be understood knowing how things that we think of as housekeeping functions - whether it's metabolism, or protein synthesis, or whatever — work differently in different kinds of dividing somatic cells.

Mirna Kvajo: What's your take on the importance of specific environments for stem cell function?

Dr. Morrison: Stem cells, we think, do depend on highly specialized microenvironments that we refer to as niches for their maintenance. A major interest in my laboratory is to understand both local signals from the niche that maintain stem cells (particularly blood-forming stem cells), as well as long-range signals. We want to understand the mechanisms that integrate stem cell function across different tissues. When you have a full-body physiological change, like starvation or pregnancy, stem cells in all different tissues have to be reading from the same song sheet. You can't have stem cells making it up as they go along in different tissues. There have to be mechanisms that integrate stem cells function across different tissues with full-body physiological changes. Estrogen and leptin are two examples of factors that do that, at least in certain stem cell systems.

We're also trying to better understand the local microenvironment that maintains stem cells. A lot of the growth factors that we know of - and that are used in regenerative medicine-either are so highly and broadly expressed that you can purify them from urine or are members of a big growth factor family that can be identified by homology with other family members (e.g., BMPs, WNTs, TGF- $\beta$ s, notch ligands). Even though we've identified scores of growth factors, there are certain things that we still can't control in terms of regenerative medicine. We still can't really grow hematopoietic stem cells in culture, which limits our ability to perform bone marrow transplants in patients. We still only have one FDA-approved anabolic growth factor to promote the formation of new bone in osteoporosis. This is a huge unmet medical need. We're looking at bone marrow to see whether there are additional growth factors that haven't been identified yet, that are highly restricted in their expression to specialized microenvironments like niches, and that explain some of the biology that we can't control. We're in the process of characterizing some of these growth factors now. Hopefully, some of them will be useful in the context of regenerative medicine.

Mirna Kvajo: When you think of stem cells as a potential source of tumor cells, do they also need the right environment to create a tumor?

Dr. Morrison: We go back and forth between trying to understand mechanisms that regulate normal stem cell self-renewal versus the self-replication of cancer cells. On one hand, cancer cells are influenced by their environment in lots of different ways. That can have important consequences on which cancer cells are actually fated to expand and which cells are not and which cancer cells are therapy-resistant. On the other hand, it's important to keep in mind that cancer cells are less factordependent than normal stem cells. People talk about niches for cancer stem cells, and there are already some compelling data to support that, but I don't think that cancer cells are going to show the same obligate dependence on highly specialized microenvironments as normal stem cells. In the end, we know that cancer cells are invasive and that they overgrow tissues. When you get a breast cancer cell, or a melanoma cell, or a prostate cancer cell in the bone marrow, they don't only grow in their restricted microenvironments where normal stem cells grow. Eventually, they ablate the structure of the tissues and they grow everywhere. It's clearly worthwhile to study the microenvironmental mechanisms that regulate cancer cells, but it will probably be more of a facultative or situational dependence than what we see with normal stem cells.

Mirna Kvajo: Like most fields, work on stem cells has been limited by the available technology. When you think about what needs to happen to move your research to the 
next level, do you have a wish for a specific new technique that would make your life easier?

Dr. Morrison: It would be nice to have a PCR for proteins. It would change the world if we could amplify proteins, and not just genes. Beyond that, it's technique by technique. Throughout my career, we've always had to scale down every technique that we work with. It's usually not hard. Usually, people use 10 million cells because they have 10 million cells, not because they need 10 million cells. If you're just a little more careful, it's amazing how easy it is to scale down a lot of things. Right now, we want to study metabolism in small numbers of highly purified cells, and really understand how metabolic pathways are used differently by different kinds of dividing somatic cells in the same tissue. We've collaborated with Ralph DeBerardinis at UT Southwestern to scale down metabolomics. We're doing metabolomics in 10,000 cells and discovering new pathways that regulate stem cell function. 


\section{$\$_{\text {CSH\& }}^{\infty}$ Cold Spring Harbor Symposia SYMPOSIA}

\section{A Conversation with Sean Morrison}

Cold Spring Harb Symp Quant Biol 2016 81: 331 -333 originally published online January 25, 2017

Access the most recent version at doi:10.1101/sqb.2016.81.031609
Creative This article is distributed under the terms of the
Commons http://creativecommons.org/licenses/by-nc/4.0/, which permits reuse and
License redistribution, except for commercial purposes, provided that the original author and source are credited.

Email Alerting Receive free email alerts when new articles cite this article - sign up in Service the box at the top right corner of the article or click here. 